\title{
Reoperative techniques and management in Hirschsprung disease: a narrative review
}

\author{
Farokh R. Demehri^, Belinda H. Dickie \\ Department of Surgery, Boston Children's Hospital, Boston, MA, USA \\ Contributions: (I) Conception and design: All authors; (II) Administrative support: None; (III) Provision of study materials or patients: None; (IV) \\ Collection and assembly of data: All authors; (V) Data analysis and interpretation: All authors; (VI) Manuscript writing: All authors; (VII) Final \\ approval of manuscript: All authors \\ Correspondence to: Farokh R. Demehri, MD. Department of Surgery, Boston Children's Hospital, 300 Longwood Ave, Fegan 3, Boston, MA 02115, \\ USA. Email: farokh.demehri@childrens.harvard.edu.
}

\begin{abstract}
The majority of children who undergo operative management for Hirschsprung disease have favorable results. A subset of patients, however, have long-term dysfunctional stooling, characterized by either frequent soiling or obstructive symptoms. The evaluation and management of a child with poor function after pull-through for Hirschsprung disease should be conducted by an experienced multidisciplinary team. A systematic workup is focused on detecting pathologic and anatomic causes of pullthrough dysfunction. This includes an exam under anesthesia, pathologic confirmation including a repeat biopsy, and a contrast enema, with additional studies depending on the suspected etiology. Obstructive symptoms may be due to technique-specific types of mechanical obstruction, histopathologic obstruction, or dysmotility—each of which may benefit from reoperative surgery. The causes of soiling symptoms include loss of the dentate line and damage to the anal sphincter, which generally do not benefit from revision of the pull-through, and pseudo-incontinence, which may reveal underlying obstruction. A thorough understanding of the types of complications associated with various pull-through techniques aids in the evaluation of a child with postoperative dysfunction. Treatment is specifically tailored to the patient, guided by the etiology of the patient's symptoms, with options ranging from bowel management to redo pull-through procedure. This review details the workup and management of patients with complications after pull-through, with a focus on the perioperative management and technical considerations for those who require reoperation.
\end{abstract}

Keywords: Hirschsprung disease; aganglionosis; pull-through; reoperation

Received: 18 May 2020; Accepted: 12 August 2020; Published: 25 July 2021.

doi: $10.21037 / \operatorname{tgh}-20-224$

View this article at: http://dx.doi.org/10.21037/tgh-20-224

\section{Introduction}

The first description of the clinical entity later termed congenital megacolon, including a description of a palliative colostomy for the disease, dates back over two millennia via Sanskrit text (1). Härold Hirschsprung, after whom the disease is now named, provided the first modern description of congenital aganglionosis and its clinical features in 1886 (2).
Hirschsprung disease (HD) manifests as a functional neonatal distal bowel obstruction due to the absence of ganglion cells in the myenteric and submucosal plexi of the distal intestinal tract. A surgical approach that addressed the pathologic etiology of HD was first described by Orvar Swenson and Alexander Bill in 1948 (3). The "Swenson pull-through" involves a full-thickness resection of the aganglionic segment and re-anastomosis of normally

^ ORCID: 0000-0002-9662-8470. 
Table 1 Differential diagnosis of obstructive and soiling symptoms after pull-through for HD (adapted from Langer et al., 2017 and Saadai et al., 2019)

\begin{tabular}{|c|c|}
\hline Symptom & Diagnoses \\
\hline \multicolumn{2}{|l|}{ Soiling symptoms } \\
\hline \multirow[t]{2}{*}{ Pseudo-incontinence } & Overflow incontinence \\
\hline & Hypermotility \\
\hline \multirow[t]{2}{*}{ Abnormal sensation } & Damage to the dentate line \\
\hline & Neo-rectum insensitivity \\
\hline \multirow[t]{2}{*}{ Damaged sphincter } & Over-stretch during pull-through \\
\hline & Prior sphincterotomy or myectomy \\
\hline \multicolumn{2}{|l|}{ Obstructive symptoms } \\
\hline \multirow[t]{5}{*}{ Mechanical obstruction } & Anastomotic stricture \\
\hline & Twisted pull-through \\
\hline & Obstructing muscular cuff \\
\hline & Obstructing Duhamel pouch \\
\hline & Postoperative adhesions \\
\hline \multirow[t]{2}{*}{ Pathologic obstruction } & Aganglionic pull-through \\
\hline & Transition zone pull-through \\
\hline $\begin{array}{l}\text { Internal anal sphincter } \\
\text { achalasia }\end{array}$ & - \\
\hline \multirow[t]{2}{*}{ Hypomotility } & Focal hypomotility \\
\hline & Diffuse hypomotility \\
\hline Functional megacolon & Stool holding behavior \\
\hline
\end{tabular}

$\mathrm{HD}$, Hirschsprung disease.

ganglionated bowel to the anal canal above the dentate line. Over the subsequent decades, several modifications to this approach have been made. The most commonly performed operations for HD are the full-thickness Swenson pullthrough, the modified Soave endorectal pull-through, and the Duhamel retrorectal pull-through; each with the option of laparoscopic assistance. While individual studies have attempted to demonstrate differential outcomes based on surgical technique, no single approach has definitively been shown to be superior (4-8). Indeed, most children have favorable long-term results. Some children, however, present with dysfunctional stooling, even in the absence of any apparent early postoperative complication. These patients require a thorough and systematic workup to identify the etiology of their dysfunctional pull-through, and for those that require reoperation, a technically sound operation is paramount. This narrative review outlines the workup and management of patients with dysfunctional stooling after pull-through for $\mathrm{HD}$, focusing on published reports from the past 20 years.

We present the following article in accordance with the Narrative Review reporting checklist (available at http:// dx.doi.org/10.21037/tgh-20-224).

\section{Dysfunctional stooling after pull-through}

The goal of resecting the aganglionic bowel in HD is to allow a child to be continent of stool while avoiding significant distension or enterocolitis. While the results of pull-through for HD have generally been regarded as favorable, with only a minority of patients requiring reoperation (9-11), recent studies have demonstrated that a larger portion of patients have long-term morbidity and problematic bowel function (12). A recent long-term quality of life study by Bjørnland et al., involving 200 patients over 4 years of age revealed that only $37 \%$ of patients reported absolutely no impaired bowel function, with $17 \%$ requiring enemas for social continence, which appeared to improve with age (13). Another European study of long-term quality of life similarly revealed that constipation and soiling persist in a substantial number of older children and adults with surgically corrected HD (14). Interestingly, adults had higher rates of straining and incomplete evacuation than children, but lower rates of soiling. Even in the shortterm, recent data suggests a high burden of poor stooling function for postoperative HD patients, with a 36\% 1-year readmission rate for noted in a US nationwide analysis by Quiroz et al. (15).

Given the variable function of patients after pull-through for HD, it is critical to employ a systematic approach to the workup of a patient with stooling issues in order to identify those who may benefit from additional operative versus nonoperative interventions. This evaluation may occur on a young child soon after pull-through, or in an older patient with ongoing issues into adulthood (16). Patients with a dysfunctional pull-through can be divided into those with obstructive symptoms (such as constipation or enterocolitis) and those with non-obstructive symptoms (such as soiling or incontinence) (Table 1). Each of these symptom categories carries a distinct differential diagnosis. The American Pediatric Surgical Association Hirschsprung Disease Interest Group has recently released guidelines outlining algorithms for the workup and management of children with obstructive symptoms (17) and frequent soiling (18). 
The various etiologies leading to the dysfunctional pullthrough are summarized in Table 1 . This review outlines the principles in evaluation that can be used to identify the specific etiology, and the therapeutic options for each case.

\section{Diagnostic evaluation}

In evaluating a child for dysfunctional stooling after repair for HD, a thorough and meticulous history and physical exam is required. The history should focus on the patient's neonatal history, other congenital anomalies or syndromic issues, initial presentation, timing and type of repair, and any recognized postoperative complications. It is helpful to document-and if possible, re-evaluatetissue pathology from the patient's prior biopsies and/or pull-through specimen. This information can offer insight into the adequacy of the prior operation, and guide next steps. In light of this past history, the clinician should seek a detailed understanding of the child's current bowel function. This includes the patient's diet, stooling pattern, toilet training status, and any history of bloating, vomiting, straining, soiling, or frank incontinence. These symptoms will help distinguish the child with obstructive versus nonobstructive symptoms. In addition, any interventions or bowel management strategies that have been implemented should be recorded-including laxatives, stool softeners, dilations, or irrigations. Often a parent will be able to report the frequency of "blowouts" suggesting enterocolitis episodes (19).

The physical exam should focus on overall nutritional status, degree of abdominal distension, and a detailed rectal exam. In the clinic, one might note a malnourished appearing child, or a distended abdomen with palpable stool—suggesting an obstructive picture. The rectal exam, starting with visual inspection, may reveal a patulous anus or diaper dermatitis-signs of incontinence. For the older child who is toilet trained, a quick examination of the child's undergarments might reveal stains from fecal incontinence. With a careful digital rectal exam, one should note rectal tone-with increased tone expected even in appropriately repaired HD, and diminished tone suggesting damage to the sphincter or anal canal. Palpation of the anastomosis may reveal a stricture, and any upstream fecal loading suggests an obstructive picture, or overflow incontinence/ encopresis in the child presenting with soiling. Occasionally, a long firm narrowing around the anastomosis, or a thickened palpable scar posterior to the anal canal, might suggest a prior anastomotic leak. Finally, on withdrawal of the examining digit, a rush of gas or stool might suggest an obstructive picture or enterocolitis.

All patients being evaluated for dysfunctional stooling after a pull-through should undergo a water-soluble contrast enema $(20,21)$. This will help outline the anatomy of the pull-through segment, and may detect a stricture, a twisted pull-through, extrinsic obstruction (i.e., a Soave cuff or dilated Duhamel pouch), or upstream dilation of the bowel. It is critical that the contrast enema is performed by a radiologist with experience in evaluating postoperative HD patients, as the enema should be administered distal to the prior anastomosis in order to fully visualize the pullthrough segment. An improperly performed enema may miss a stricture or obstructing Soave cuff if the catheter is placed too deep. A simple abdominal plain film is also helpful to evaluate for bowel dilation and fecal loading. Additional imaging such as computed tomography (CT) or magnetic resonance imaging (MRI) is not routinely necessary.

\section{Exam under anesthesia (EUA)}

To complete the initial evaluation, all patients should undergo an examination under anesthesia, along with anorectal manometry (ARM), and rectal biopsy. First, ARM is performed prior to the induction of anesthesia, aside from a mild anxiolytic. ARM can help document the absence of a recto-anal inhibitory reflex (RAIR), as expected in any patient with HD even after successful repair, and may also detect decreased sphincter tone or more proximal rectal manometric abnormalities (Figure 1). After ARM is complete, the patient may undergo EUA. This allows for a detailed assessment of the integrity of the anal canal, the location of the anastomosis relative to the dentate line, presence of a stricture, or the presence of extrinsic compression such as an obstructing Soave cuff. This starts with a visual exam, assessing for baseline prolapse or skin changes and whether the anus is open or closed. Then, a Lone Star (CooperSurgical, Trumbull, CT, USA) retractor may be placed to expose the anal canal. This provides exposure of the transitional epithelium and allows circumferential inspection of the dentate line. The hooks of the retractor should be set distally, near the muco-epidermal junction, to fully expose the anal canal and the dentate line. The anastomosis should be just proximal to the dentate line. The examiner can document its position relative to the dentate line.

The anesthetic episode may conclude with a full 


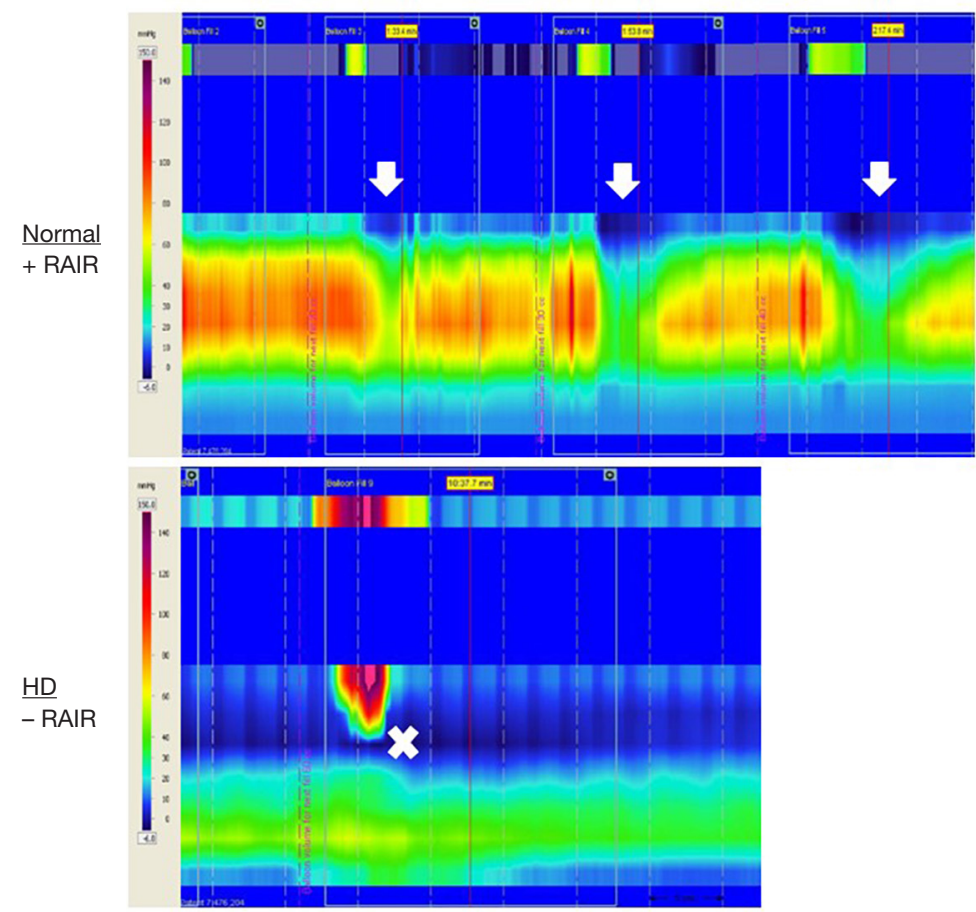

Figure 1 Anorectal manometry (ARM) tracings demonstrating the presence of a recto-anal inhibitory reflex (RAIR), wherein the pressure within the internal anal sphincter decreases (arrows) with increasing pressure applied to the rectal balloon. In a patient with Hirschsprung disease (HD), there is an absence of anal relaxation despite high rectal balloon pressure (x) (courtesy of Claudio J. Morera, MD, Boston Children's Hospital).

thickness rectal biopsy. This is performed at the posterior midline just above the level of the prior anastomosis. In addition, rigid or flexible endoscopy may be performed if there is concern for more proximal pathology such as a twisted pull-through, or if the patient had a prior Duhamel reconstruction. This allows thorough assessment of the proximal pull-through segment or Duhamel pouch, assessing for obstruction, dilation, or a blind-ending pouch. Finally, if there is concern for dysmotility based on colonic dilation-as further described below-one may perform colonic manometry during the same session. By the conclusion of this examination, the surgeon has assessed for anatomic or pathologic causes of stooling dysfunction after pull-through for HD.

\section{The soiling patient}

As summarized in Table 1, the causes of soiling or incontinence after repair for HD include pseudoincontinence, abnormal sensation, and damaged sphincter. Pseudo-incontinence is the most common cause of soiling in children with $\mathrm{HD}$, and is defined as soiling despite intact sensation and sphincter function (18). The water-soluble contrast enema and colonic manometry will help distinguish those with pseudo-incontinence due to obstruction and overflow-which will manifest as a dilated and dysmotile colon - and those due to intrinsic hypermotility - which will manifest as a hypermotile and nondilated colon. The etiology of hypermotility in HD is thought to be due to high amplitude propagating contractions in the colon which are unopposed by the anal sphincter $(22,23)$. This is important to identify, as these children should be treated with a constipating diet, avoiding laxatives, and potentially anti-motility medications-contrary to many other patients with HD. Those with pseudo-incontinence due to an obstructive mechanism may present with distension and fecal loading on exam. This overflow incontinence requires treatment of their underlying obstructive pathology, as outlined below.

The remaining causes of soiling are considered true incontinence-and are defined by true loss of anal sphincter control or loss of sensation. Unfortunately, there are no 

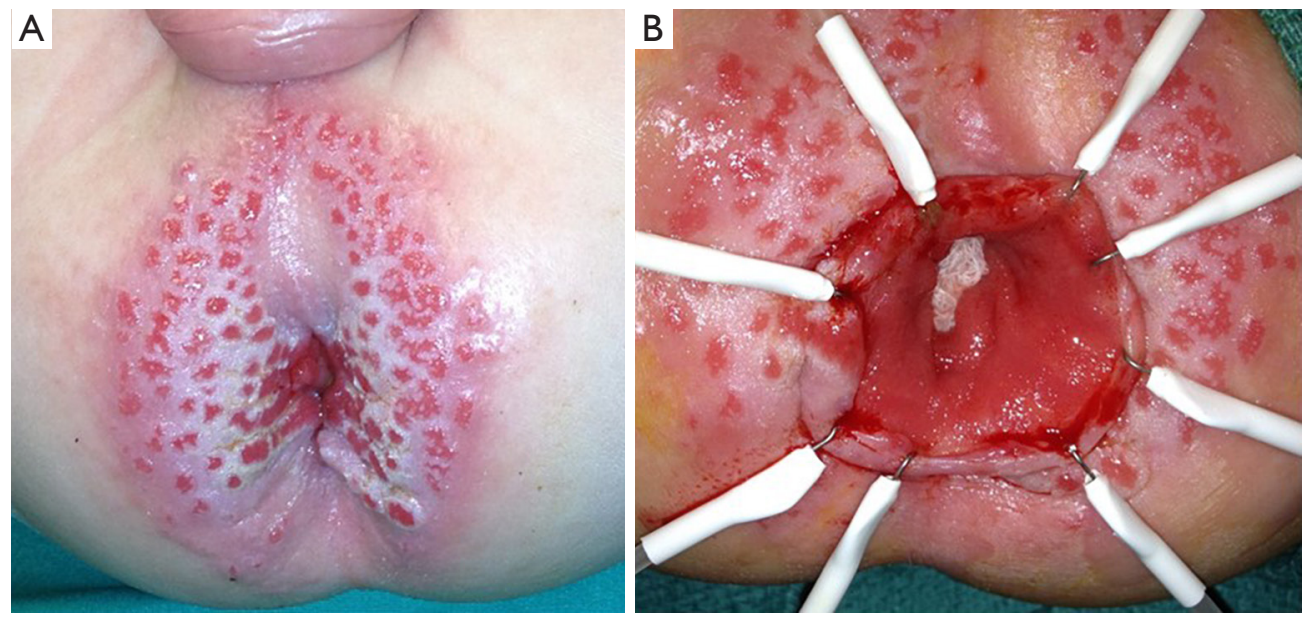

Figure 2 A boy with fecal incontinence after pull-through for HD. Exam under anesthesia reveals (A) severe diaper dermatitis and (B) destruction of the dentate line from the initial pull-through, with colonic mucosa from the pull-through reaching the skin and loss of the anal canal. HD, Hirschsprung disease.

durable current surgical treatments to remedy these issues besides bowel management with enemas-so it is critical for the surgeon to avoid these iatrogenic injuries at the initial operation. The normal sensation of the rectum relies on sensing distension of the rectum, and on the sensitive transitional epithelium defined by the dentate line. The anastomosis for pull-through for HD should be performed $5-15 \mathrm{~mm}$ above the dentate line. Damage to the dentate line may render a patient insensate and therefore incontinent. A recent study by De la Torre $e t$ al. demonstrated universal fecal incontinence in patients with damage to the anal canal with pull-through, while those with an intact anal canal were fecally continent though at a higher risk of colitis (24). Figure 2 depicts a male patient who presented with severe diaper dermatitis suggesting chronic soiling (A). On EUA, his anastomosis was found to be below the level of the dentate line, with loss of the anal canal's transitional epithelium (B). This patient responded well to an antegrade enema program.

The remaining group of patients with true incontinence after pull-through for HD are those with a damaged sphincter. This may be due to secondary intentional damage to the sphincter via myectomy-a technique sometimes employed to treat recurrent Hirschsprung Associated Enterocolitis (HAEC), which will be discussed later. It may also be the result of circumferential over-stretching of the sphincter during the initial pull-through procedure (25). Figure 3 depicts a patient with frank fecal incontinence who has a patulous, atonic anal sphincter (Figure $3 A$ ). On inspection, the dentate line is largely intact (Figure 3B), but without an intact sphincter, the patient remained incontinent and was treated with enemas. This patient's primary operation was a fully transanal endorectal pullthrough. It is possible that the transanal approach only may increase the risk of over-stretching the sphincter unless exquisite care is taken during retraction (26). The use of laparoscopy to perform the rectal dissection may reduce the amount of time performing a transanal resection, potentially reducing the risk of sphincter damage (27). A novel technique of anal canal plasty for patients with overstretched sphincter after transanal pull-through described by Yasui et al. may improve function in selected patients (28). In all, the majority of patients with true incontinence after pullthrough-due to either damage to the anal canal mucosa or to the muscular sphincter mechanism-will require enema therapy for social continence. Antegrade continence enemas offer a favorable quality of life, and for patients with $\mathrm{HD}$ in particular, some will obtain recovery of continence and ability to wean off of enemas (29). Another promising option for social continence is the Peristeen integrated transanal irrigation system (Coloplast, Denmark), which may allow highly compliant children to avoid a surgical antegrade enema access procedure while providing a favorable quality of life (30).

\section{The obstructed patient}

The differential diagnosis for the patient presenting with 

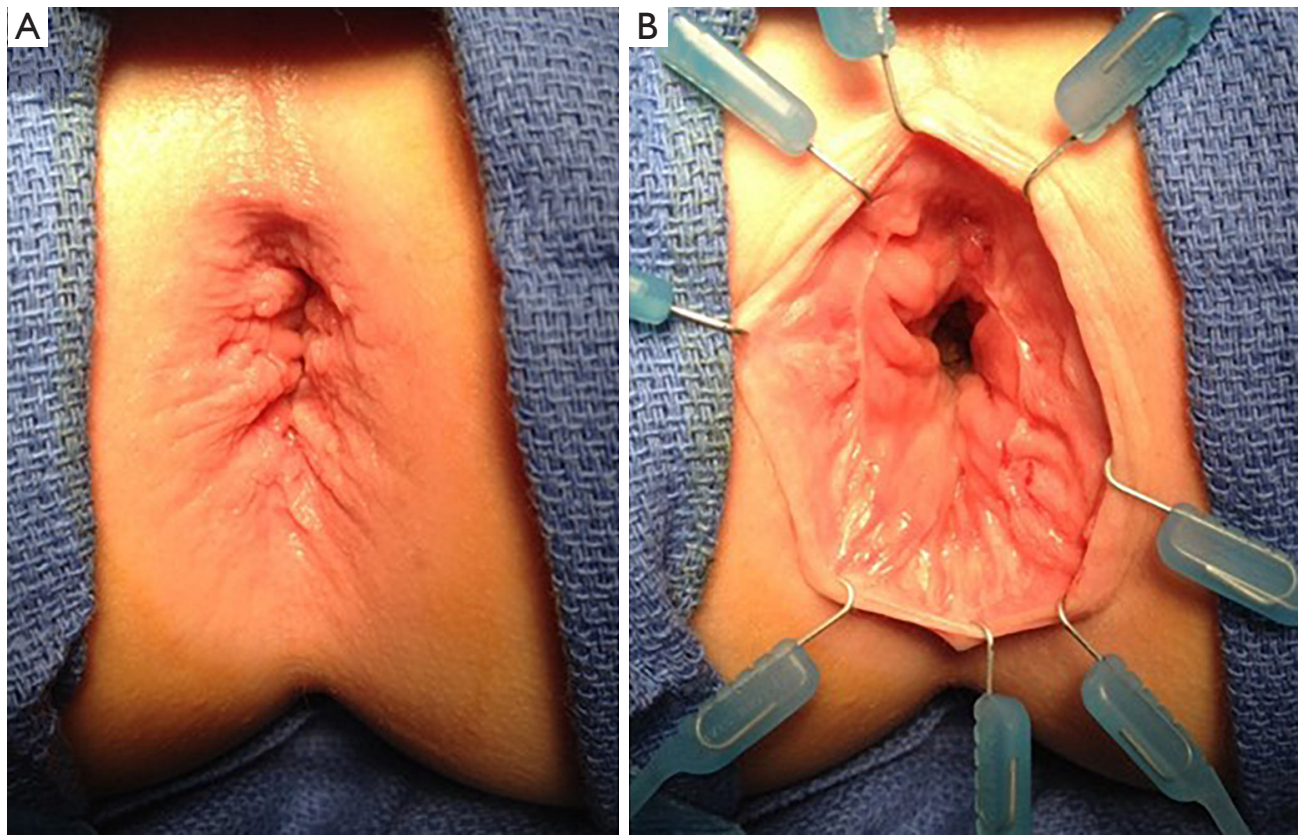

Figure 3 A child with soiling after pull-through for HD. Exam under anesthesia reveals a patulous anus (A) in the setting of a preserved dentate line (B), suggesting over-stretch and damage to the anal sphincter. HD, Hirschsprung disease.

obstructive symptoms after definitive surgery for HD is summarized in Table 1 (18). Some children may present with symptoms that are essentially unchanged from prior to their pull-through. Others may have more severe obstructive symptoms, or they may present with soiling in the setting of pseudo-incontinence. The severity of the obstructive symptoms may range from the child who requires oral bowel management medications to the patient who presents with recurrent HAEC (31). Indeed, the diagnosis of HAEC after a successful pull-through should elicit a thorough evaluation for obstructive etiology (32). While many factors have been implicated in the pathophysiology of HAEC, including age at diagnosis, genetics, and the gut microbiome, an obstructive process much be ruled out as a potentially reversible cause $(33,34)$. By employing the systematic evaluation outlines above, beginning with careful physical exam and contrast enema, the surgeon should distinguish between mechanical and non-mechanical (or functional) obstruction. For those patients in whom mechanical obstruction has been ruled out, a full-thickness rectal biopsy is necessary to then rule out histopathologic obstruction, such as pull-through of bowel that is aganglionic, within the transition zone, or more rare and controversial diagnoses such as acquired aganglionosis, skip area, or intestinal neuronal dysplasia type B $(35,36)$. For a child with obstructive symptoms who has a non-obstructive contrast enema and normal rectal biopsy, the remaining elements in the differential include hypomotility, internal anal sphincter achalasia, and functional megacolon. A trial of botulinum toxin injection and motility workup can help differentiate among these causes.

\section{Mechanical obstruction}

The first etiology of obstructive symptoms post-pullthrough that should be ruled out is mechanical obstruction. This is usually evident based on knowledge of the type of prior operative technique and the findings on physical exam and contrast enema. A child with an anastomotic stricture may undergo a trial of serial dilations, though this is most successful in very short strictures $(<1 \mathrm{~cm})$ that are diagnosed early ( $<6$ weeks). The use of topical mitomycin-C to increase the efficacy of dilation has been reported (37). Longer strictures suggest ischemia of the pull-through segment, or anastomotic leak leading to inflammatory scarring of the segment, which is unlikely to resolve with dilations. For these patients, a redo pull-through is required (10). Our approach is to perform a Swenson procedure, resecting the full thickness prior anastomosis via the transanal route, using laparoscopy as to gain length on the proximal bowel 


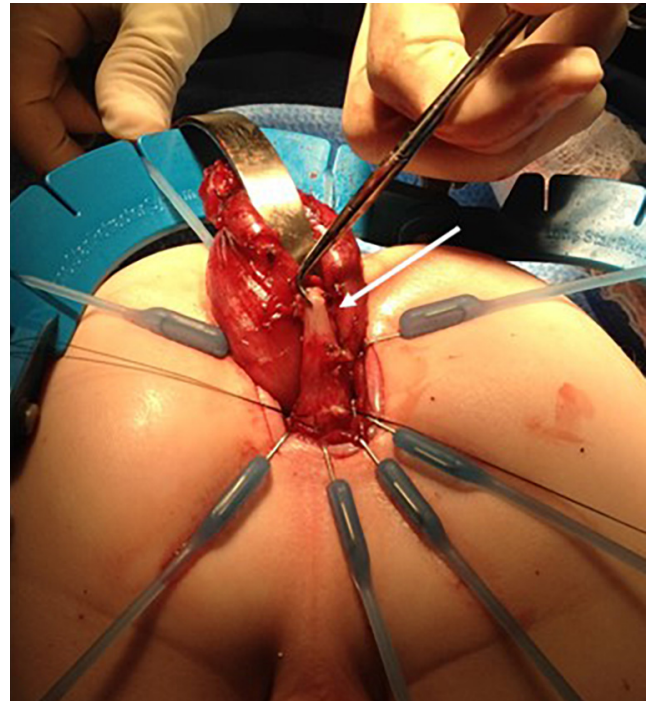

Figure 4 A restricting aganglionic cuff (arrow) after a Soave endorectal pull-through.

as needed. An alternative approach, described by Langer, is to convert such patients to a Duhamel by taking advantage of the untouched presacral plane and stapling across the stricture while creating the Duhamel anastomosis (38).

A twisted pull-through, if evident in the immediate postoperative period as an acute obstruction, should undergo prompt revision. For the rare patient who has an apparently uncomplicated postoperative course but present with chronic obstructive symptoms, a partially obstructing twist may be evident by a contrast enema showing a tapering or waist in the pull-through above the anastomosis. At the index operation, maneuvers to decrease the risk of rotating the bowel include applying serial sutures at the 12 o'clock position to maintain orientation and advancing a flexible catheter such as a Foley retrograde through the pull-through segment to ensure no resistance as it navigated a twisted/obstructed lumen. The use of laparoscopy is helpful to visually confirm a straight pull-through as well. If a twist is suspected in a postoperative patient based on contrast study, a flexible sigmoidoscopy can be used to confirm the diagnosis. Once confirmed, this requires a redo pull-through operation, ideally with transabdominal/ laparoscopic assistance to ensure proper orientation of the bowel.

\section{The obstructing Soave cuff}

An understanding of the technique used for the patient's index operation is critical in order to detect techniquespecific causes of mechanical obstruction. For a patient who has had a Soave endorectal pull-through, a well-established complication is an obstructing muscular cuff $(18,20)$. To review, the Soave technique involves a submucosal dissection of the distal rectum to theoretically preserve the pelvic splanchnic nerves, leaving an aganglionic muscular cuff of varying length depending on surgeon preference. This muscle is typically split or partially resected in the posterior midline to theoretically decrease the risk of re-adherence and obstruction due to this high resistance cuff (16). Despite these maneuvers, however, this aganglionic tissue can be problematic in certain patients. The surgeon may appreciate the obstructive muscular cuff during rectal exam as a ridge of tissue posteriorly that rolls between the examiners finger and the sacrum. It may be visible on a contrast study as a posterior indentation on the distal pullthrough or widened presacral space.

The surgical options for this include posterior myotomy/ myectomy (POMM), or redo pull-through with excision of the aganglionic cuff $(11,39)$. The POMM involves dividing or resecting a portion of the posterior aganglionic cuff via a transanal approach. This may be performed in a similar fashion as a full-thickness rectal biopsy, however ensuring that the excision includes the full thickness of the pullthrough bowel as well as the full thickness muscular cuff deep to this, and the intervening scar tissue. It is important to rule out concomitant residual aganglionosis above the anastomosis prior to this, however, as these patients uniformly require redo pull-through. The redo operation here involves resecting the aganglionic cuff as well as the pull-through segment, converting the patient to a Swensontype pull-through, as shown in Figure 4, where a densely adherent portion of the muscular cuff is marked. Some authors advocate preserving the anterior portion of the Soave cuff, and resecting the posterior half-a so-called "Soaveson"-but this may be more challenging as the plane between the muscular cuff and the pull-through segment is often quite scarred (16).

\section{The problematic Dubamel}

In patients with a history of Duhamel reconstruction, a unique type of obstructive pathology is the obstructing Duhamel pouch. To review, the Duhamel technique involves preserving the distal aganglionic rectum, and bringing the ganglionic pull-through segment posterior to this in order to create a side-to-side anastomosis between the two, often 

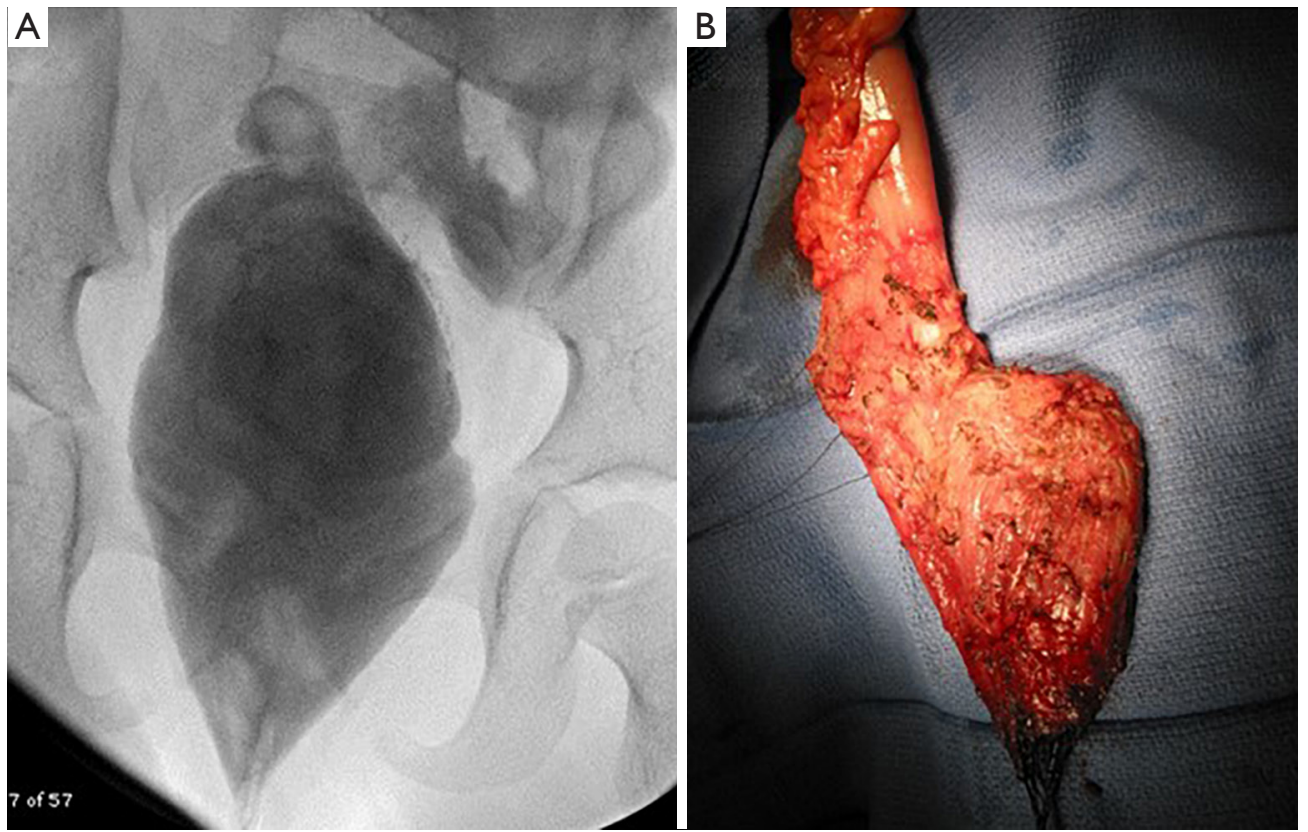

Figure 5 A child with recurrent enterocolitis after a Duhamel operation. Contrast enema demonstrates a dilated "mega pouch" (A), requiring resection of the pouch (B) with redo Swenson pull-through.

with a linear stapler fired trans-anally, versus a handsewn anastomosis. This creates a composite wherein the posterior portion of the reconstructed rectum has ganglion cells, and the anterior portion is the aganglionic original rectum. While this technique has high rates of success worldwide, it does generate unique complications related to this intentionally retained aganglionic bowel (40). First, if the side-to-side anastomosis does not incorporate the entire length of the aganglionic rectum, a "spur" of aganglionic bowel above the anastomosis may be left behind. This can dilate and lead to intermittent obstruction of the pullthrough segment due to extrinsic compression. Diagnosis is confirmed via endoscopy, which will demonstrate a blindending dilated limb, and on contrast enema which will demonstrate the same. Options for addressing this include laparoscopic or open resection via the trans-abdominal route, or extension of the prior linear anastomosis using a linear stapler via the trans-anal route, thus incorporating this "spur" into the proper composite Duhamel pouch (38).

The other complication of a Duhamel reconstruction that should be considered is the "mega pouch"—where the composite pouch becomes progressively dilated and dysfunctional. These patients may present with soiling due to pseudo-incontinence, impaction, or enterocolitis. In a series of patients requiring reoperation after Duhamel by
Chatoorgoon et al., this was the most common indication for redo pull-through (41). These patients may initially be treated with aggressive bowel management and irrigations, but resection of the dilated segment is usually required (Figure 5). This generally requires a combined abdominal and transanal approach. While laparoscopy may be helpful in other types of redo pull-through, invariably the Duhamel pouch creates a dense fibrotic reaction in the presacral space requiring an open, often very difficult resection in a frozen pelvis, sometimes aided by a posterior sagittal incision (42). After resecting the pouch and any dilated upstream bowel, confirmed ganglionic bowel is pulled through for a Swenson-type reconstruction. Temporary diversion should be considered either at the time of the resection or at a prior date to decompress the pouch beforehand, especially any patient who requires a posterior sagittal incision. If performed prior to revision, this may allow the proximal bowel to be less dilated and more suitable for anastomosis at the time of redo pull-through.

\section{Histopathologic obstruction}

As outlined earlier, a key step in the workup of a patient with obstructive symptoms after pull-through is a full-thickness biopsy in order to rule out a range of 


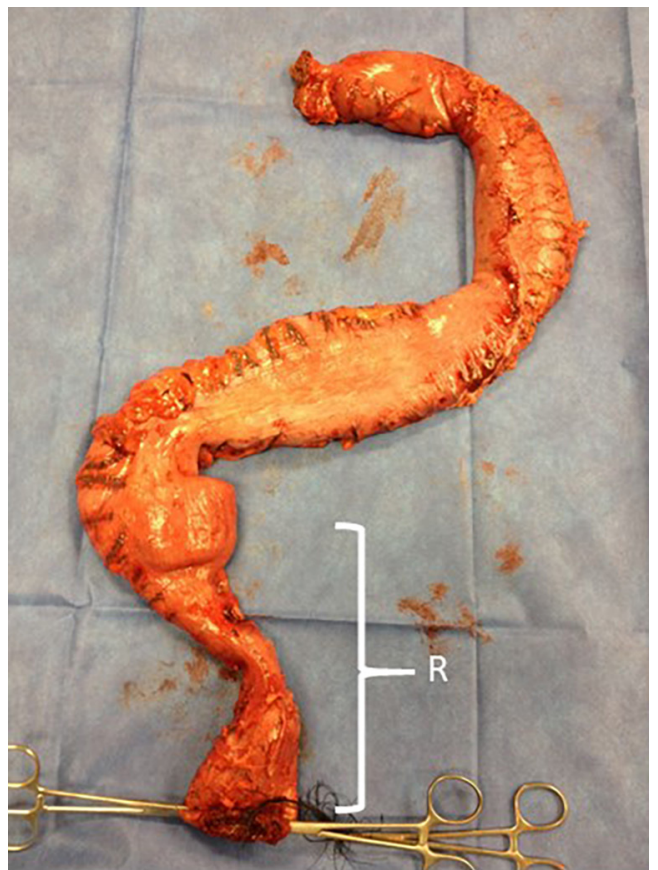

Figure 6 A child with obstructive symptoms after Swenson pullthrough was found to have a retained aganglionic segment (R), requiring redo pull-through and resection of this as well as dilated upstream bowel.

histopathologic abnormalities that may require redo pullthrough. This includes residual aganglionosis, transition zone pull-through, and more rare diagnoses such as acquired aganglionosis, skip area, and intestinal neuronal dysplasia type B. A thorough exploration of each of these etiologies has recently been conducted by Kapur et al. (35). With the potential exception of intestinal neuronal dysplasia, all forms of histopathologic abnormality above the level of the prior anastomosis require a redo pullthrough. In published series of patients who have required redo pull-through, abnormal pathology was the indication for between $35 \%$ and $63 \%$ of patients (43-46). When performing a redo pull-through for patients with abnormal pathology, it is of utmost importance to establish the correct level of normal pathology for the new anastomosis. This may be conducted via transabdominal laparoscopic or open full-thickness biopsies, or serial transanal biopsies. Recent guidelines for synoptic reporting of surgery and histology in HD by Veras et al. provide detailed recommendations on a standardized method of obtaining and labeling such leveling biopsies (47). When a level has been selected based on a ganglionic biopsy, the resection should then be performed at least $5 \mathrm{~cm}$ proximal to this in order to reduce the likelihood of a transition zone pull-through. The bowel immediately upstream of the histopathologic obstruction is usually dilated, and it is best to resect this dilated bowel which is often dysfunctional. In doing so, often the surgeon has achieved adequate proximal distance from the ganglionated biopsy. In addition, the full ring of bowel at the level of transection should undergo frozen section analysis by an experience pediatric pathologist to confirm circumferential normal bowel innervation. Rather than sectioning this ring on the field, it may be preferable for the entire specimen to be delivered to the pathologist for en face frozen section (47). With full confirmation of histologically normal bowel, an anastomosis may be created. Figure 6 demonstrates the resected specimen from a patient with residual aganglionosis, including the significant length of upstream dilated bowel.

\section{Internal anal sphincter achalasia}

For the patient with no evidence of mechanical obstruction on exam or contrast study, and normal pathology on biopsy, the remaining differential includes internal anal sphincter achalasia, dysmotility, and stool holding behavior (Table 1). At the time of the EUA and repeat rectal biopsy, the performance of ARM can help differentiate between these entities, as a high resting pressure suggests achalasia in the setting of a normal biopsy. If a patient is undergoing EUA with ARM and repeat biopsy with a relatively normal contrast study, a trial of botulinum toxin injection into the internal anal sphincter may be performed during the same anesthetic episode. If there is symptomatic improvement to this maneuver, the patient may be treated with repeat injections for internal anal sphincter achalasia. If there is no response, the remaining workup should focus on differentiating between dysmotility and functional megacolon.

Internal anal sphincter achalasia is thought to represent a variant of anal sphincter spasm, as a hypertonic sphincter is expected in patients with $\mathrm{HD}$ due to abnormal innervation (48). The majority of patients will respond well to bowel management and time, as they age and develop the ability to generate adequate intra-abdominal pressure to push through the hypertonic sphincter. If symptoms persist beyond a year of age, or if symptoms are severe including enterocolitis episodes, repeat botulinum toxin injection is an effective therapeutic option (49). Botulinum toxin injection may be performed by direct palpation or ultrasound 


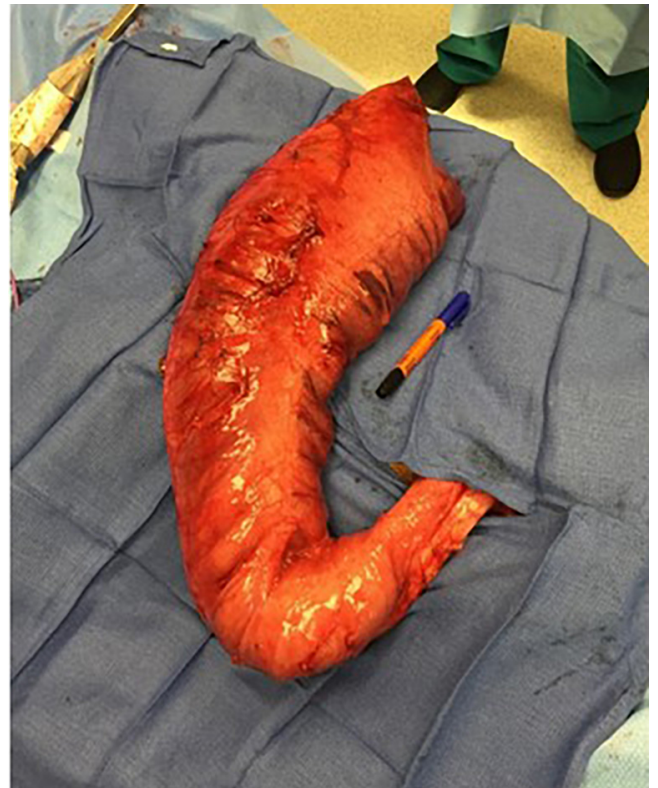

Figure 7 A focally dilated and dysmotile left colon in a child with a histopathologically normal pull-through and ongoing obstructive symptoms not responsive to botulinum toxin.

guidance, which may improve effectiveness by allowing direct visualization of the internal anal sphincter (50). The surgeon may inject between 50 and $100 \mathrm{U}$ of botulinum toxin $\mathrm{A}(3-5 \mathrm{mg} / \mathrm{kg})$ diluted in $1.0 \mathrm{~mL}$ of saline, in the plane between the internal and external anal sphincter, inserting the needle at the anal verge to beyond the dentate line and injecting during withdrawal. This is repeated to divide the dose through multiple injections, avoiding the urethra anteriorly.

While the response to botulinum toxin is transient, lasting about three months, it may be enough to allow symptom relief until the child's obstructive symptoms improve with time. In resource poor settings where botulinum toxin may be unavailable, topical nitroglycerin paste is an alternative (51). For the patient who had a prior Soave endorectal pull-through, a posterior myectomy may provide more durable relief of anal achalasia, though this does carry the risk of fecal incontinence, so repeat botulinum toxin injections may be preferred $(52,53)$.

\section{Hypomotility and functional megacolon}

If the patient with a normal rectal biopsy and no evidence of mechanical obstruction does not respond to botulinum toxin injection to relax the anal sphincter, then a functional disorder of the colon or pelvic floor should be considered. A thorough motility workup should be conducted, using institution-specific methodologies. This may include a radioopaque marker study (54), scintigraphic colonic motility study (55), and/or colonic manometry (56). Manometry is able to differentiate pan-colonic hypomotility from segmental or focal hypomotility. This distinction is critical, as focal dysmotility in this population may benefit from resection of the dilated and dysmotile segment. As this dysmotile segment is the most distal bowel, this may be resected via a redo pullthrough (Figure 7), though longitudinal tapering without resection has been described (57).

For those patients with pan-colonic hypomotility, and those with normal motility studies, segmental resection is not therapeutic. These patients are treated with aggressive bowel management, including colonic stimulants, laxatives, and enemas as needed. For those who have stool holding behavior and functional megacolon, pelvic physical therapy and biofeedback may be beneficial if the child is mature enough to participate effectively. Antegrade enema access may be considered, and for those who fail these interventions, ileostomy may be required. While in some cases such an ileostomy may be permanent, certain patients may respond to diversion and subsequent restoration of enteral continuity after resolution of distal colonic dilation.

A novel intervention that may be considered in these patients is neuromodulation, either via transcutaneous interferential (IF) stimulation or sacral nerve stimulation (SNS). Only IF has been studied specifically in HD, with reported improvement in constipation symptoms of $66 \%$ of patients along with behavioral therapy (58). Though SNS has not been evaluated rigorously in HD, non-controlled studies including HD patients among other etiologies of constipation report $>50 \%$ symptom improvement $(59,60)$. Future work may demonstrate an important role for neuromodulation in this population.

\section{Technical considerations}

A redo pull-through operation for HD can be technically demanding, and should be undertaken by a team that is experienced in such cases. The surgeon should be prepared for transabdominal and transanal access, with either laparoscopic or open approaches. A full body prep is helpful for redo pull-through cases, to allow full access to the abdomen in the event that further colonic mobilization is required. Laparoscopy is particularly useful as a means to perform segmental biopsies when required, and to perform 


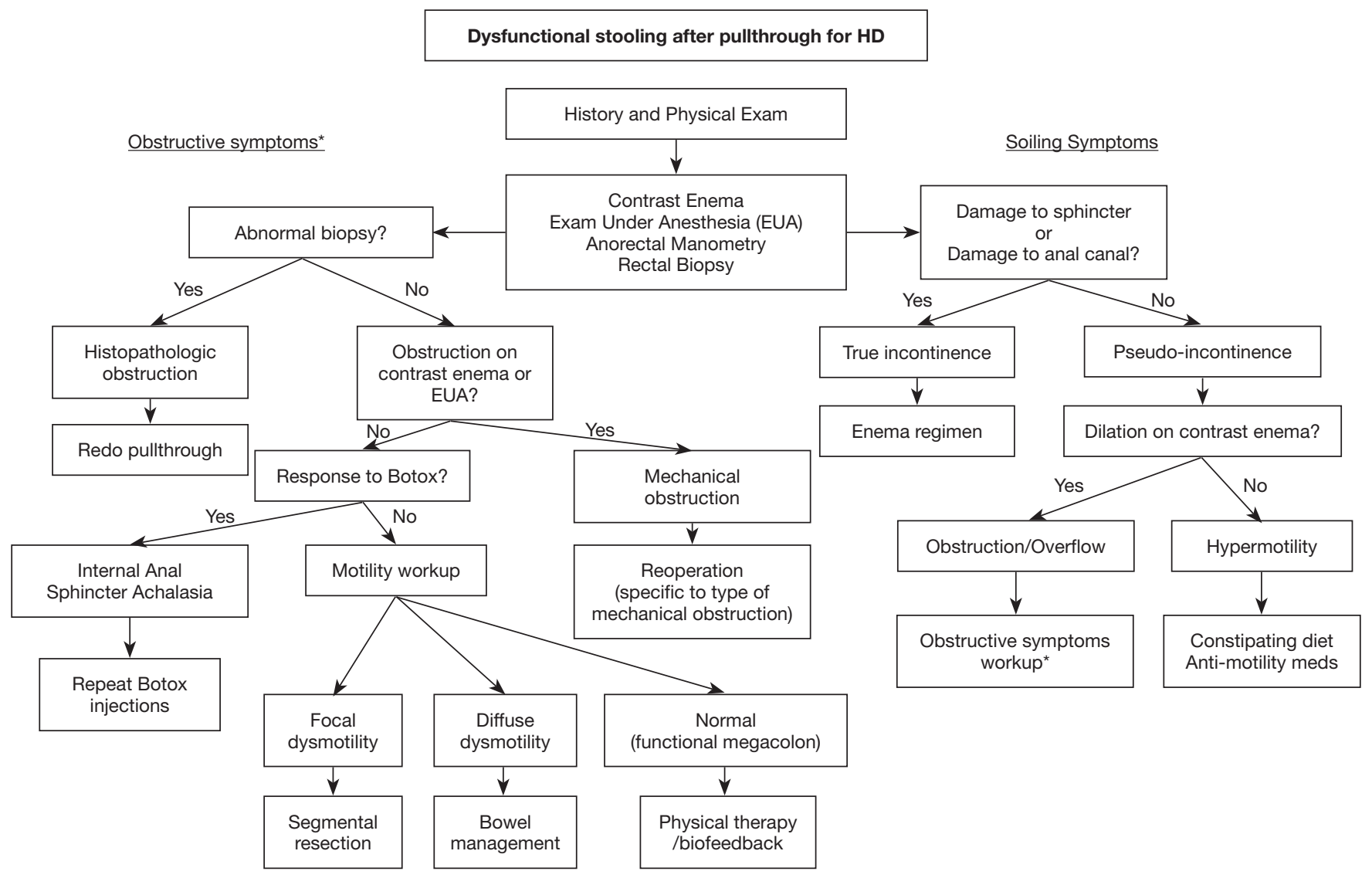

Figure 8 Management algorithm for patients with dysfunctional stooling after pull-through for Hirschsprung disease. *, a patient with soiling symptoms found to have overflow incontinence should undergo workup for obstructive symptoms.

adhesiolysis and mobilization of the proximal colon to allow a tension-free redo pull-through (46). In addition, it can be helpful to assess the level of dilation of the proximal bowel, which may require additional resection. It is critical to assess the blood supply to the mobilized bowel for pull-through, and to preserve the marginal artery so that proper perfusion is maintained. This may require a high ligation of the inferior mesenteric artery and vein to allow a mesenterypreserving mobilization of the left colon. For redo cases, especially where the more proximal colon is being pulled through, it can be challenging to assess the marginal artery-and adjunct maneuvers such as fluorescein dye may be helpful to assess perfusion prior to anastomosis (61). A helpful guide prior to pulling the bowel through is to use the pubic symphysis as a guide, expecting bowel that can reach over $2 \mathrm{~cm}$ below the pubic symphysis to have adequate reach for a pull-through.

Prior to considering a repeat pull-through procedure, each patient should undergo optimization of nutritional and functional parameters. In many patients with dysfunctional stooling from HD, malnutrition and dilation are significant operative risk factors. One should seriously consider a diverting stoma-usually ileostomy-in these patients. This allows the chronically obstructed child to achieve enteral nutritional gain while allowing the dilated colon to recover prior to definitive pull-through.

\section{Conclusions}

While most patients have satisfactory results after initial surgical treatment for HD, a subset with have ongoing dysfunctional stooling. A thoughtful and systematic evaluation (Figure 8) can help identify those patients who may benefit from nonoperative management, versus those where reoperation may be beneficial. Reported outcomes from redo pull-through for HD are variable but generally favorable. Single center series have reported incontinence rates after redo pull-through ranging from $6 \%$ to $43 \%$, 
both with endorectal pull-through as the repeat procedure of choice $(62,63)$. Future work may address whether using more novel minimally invasive techniques might improve continence rates after reoperative surgery for HD.

\section{Acknowledgments}

Funding: None.

\section{Footnote}

Provenance and Peer Review: This article was commissioned by the Guest Editors (Eduardo Perez, Samir Pandya, and Matthew S. Clifton) for the series "Current Topics in Pediatric General Surgery" published in Translational Gastroenterology and Hepatology. The article has undergone external peer review.

Reporting Checklist: The authors have completed the Narrative Review reporting checklist. Available at http:// dx.doi.org/10.21037/tgh-20-224

Conflicts of Interest: Both authors have completed the ICMJE uniform disclosure form (available at http://dx.doi. org/10.21037/tgh-20-224). The series "Current Topics in Pediatric General Surgery" was commissioned by the editorial office without any funding or sponsorship. The authors have no other conflicts of interest to declare.

Ethical Statement: The authors are accountable for all aspects of the work in ensuring that questions related to the accuracy or integrity of any part of the work are appropriately investigated and resolved.

Open Access Statement: This is an Open Access article distributed in accordance with the Creative Commons Attribution-NonCommercial-NoDerivs 4.0 International License (CC BY-NC-ND 4.0), which permits the noncommercial replication and distribution of the article with the strict proviso that no changes or edits are made and the original work is properly cited (including links to both the formal publication through the relevant DOI and the license). See: https://creativecommons.org/licenses/by-nc-nd/4.0/.

\section{References}

1. Raveenthiran V. Knowledge of ancient Hindu surgeons on Hirschsprung disease: Evidence from Sushruta Samhita of circa 1200-600 bc. J Pediatr Surg 2011;46:2204-8.

2. Sergi C. Hirschsprung's disease: Historical notes and pathological diagnosis on the occasion of the 100 th anniversary of Dr. Harald Hirschsprung's death. World J Clin Pediatr 2015;4:120.

3. Swenson O, Bill AJ. Resection of rectum and rectosigmoid with preservation of the sphincter for benign spastic lesions producing megacolon; an experimental study. Surgery 1948;24:212-20.

4. Aworanti OM, Mcdowell DT, Martin IM, et al. Comparative review of functional outcomes post surgery for Hirschsprung's disease utilizing the paediatric incontinence and constipation scoring system. Pediatr Surg Int 2012;28:1071-8.

5. Mao YZ, Tang ST, Li S. Duhamel operation vs. transanal endorectal pull-through procedure for Hirschsprung disease: A systematic review and meta-analysis. J Pediatr Surg 2018;53:1710-5.

6. Tannuri ACA, Ferreira MAE, Mathias AL, et al. Long-term results of the Duhamel technique are superior to those of the transanal pullthrough: A study of fecal continence and quality of life. J Pediatr Surg 2017;52:449-53.

7. Seo S, Miyake H, Hock A, et al. Duhamel and Transanal Endorectal Pull-throughs for Hirschsprung' Disease: A Systematic Review and Meta-analysis. Eur J Pediatr Surg 2018;28:81-8.

8. Parahita IG, Makhmudi A, Gunadi. Comparison of Hirschsprung-associated enterocolitis following Soave and Duhamel procedures. J Pediatr Surg 2018;53:1351-4.

9. Teitelbaum DH, Cilley RE, Sherman NJ, et al. A decade of experience with the primary pull-through for Hirschsprung disease in the newborn period: A multicenter analysis of outcomes. Ann Surg 2000;232:372-80.

10. Peña A, Elicevik $M$, Levitt MA. Reoperations in Hirschsprung disease. J Pediatr Surg 2007;42:1008-13; discussion 1013-4.

11. Ralls MW, Coran AG, Teitelbaum DH. Reoperative surgery for Hirschsprung disease. Semin Pediatr Surg 2012;21:354-63.

12. Granström AL, Danielson J, Husberg B, et al. Adult outcomes after surgery for Hirschsprung's disease: Evaluation of bowel function and quality of life. J Pediatr Surg 2015;50:1865-9.

13. Bjørnland K, Pakarinen MP, Stenstrøm P, et al. A Nordic multicenter survey of long-term bowel function after transanal endorectal pull-through in 200 patients with rectosigmoid Hirschsprung disease. J Pediatr Surg 2017;52:1458-64. 
14. Meinds RJ, van der Steeg AFW, Sloots CEJ, et al. Longterm functional outcomes and quality of life in patients with Hirschsprung's disease. Br J Surg 2019;106:499-507.

15. Quiroz HJ, Perez EA, Franklin KN, et al. Pull-through procedure in children with Hirschsprung disease: A nationwide analysis on postoperative outcomes. J Pediatr Surg 2020;5 5:899-903.

16. Calkins CM. Hirschsprung Disease beyond Infancy. Clin Colon Rectal Surg 2018;31:51-60.

17. Langer JC, Rollins MD, Levitt M, et al. Guidelines for the management of postoperative obstructive symptoms in children with Hirschsprung disease. Pediatr Surg Int 2017;33:523-6.

18. Saadai P, Trappey AF, Goldstein AM, et al. Guidelines for the management of postoperative soiling in children with Hirschsprung disease. Pediatr Surg Int 2019;35:829-34.

19. Pastor AC, Osman F, Teitelbaum DH, et al. Development of a standardized definition for Hirschsprung'sassociated enterocolitis: a Delphi analysis. J Pediatr Surg 2009;44:251-6.

20. Levitt MA, Dickie B, Peña A. Evaluation and treatment of the patient with Hirschsprung disease who is not doing well after a pull-through procedure. Semin Pediatr Surg 2010;19:146-53.

21. Garrett KM, Levitt MA, Peña A, et al. Contrast enema findings in patients presenting with poor functional outcome after primary repair for Hirschsprung disease. Pediatr Radiol 2012;42:1099-106.

22. Kaul A, Garza JM, Connor FL, et al. Colonic hyperactivity results in frequent fecal soiling in a subset of children after surgery for Hirschsprung disease. J Pediatr Gastroenterol Nutr 2011;52:433-6.

23. Di Lorenzo C, Solzi GF, Flores AF, et al. Colonic motility after surgery for Hirschsprung's disease. Am J Gastroenterol 2000;95:1759-64.

24. De la Torre L, Cogley K, Santos K, et al. The anal canal is the fine line between "fecal incontinence and colitis" after a pull-through for Hirschsprung disease. J Pediatr Surg 2017;52:2011-7.

25. Bischoff A, Frischer J, Knod JL, et al. Damaged anal canal as a cause of fecal incontinence after surgical repair for Hirschsprung disease - a preventable and under-reported complication. J Pediatr Surg 2017;52:549-53.

26. Stensrud KJ, Emblem R, Bjørnland K. Anal endosonography and bowel function in patients undergoing different types of endorectal pull-through procedures for Hirschsprung disease. J Pediatr Surg 2015;50:1341-6.
27. Bawazir OA. Laparoscopic-Assisted Transanal PullThrough in Hirschsprung Disease: Does Laparoscopic Dissection Minimize Anal Overstretching? J Laparoendosc Adv Surg Tech A 2020;30:338-43.

28. Yasui Y, Nishida S, Shironomae T, et al. Surgical approach for fecal incontinence with a patulous anus after transanal pull-through for Hirschsprung disease. J Pediatr Surg 2017;52:1070-5.

29. Chong C, Featherstone N, Sharif S, et al. 5 years after an ACE: what happens then? Pediatr Surg Int 2016;32:397-401.

30. Corbett P, Denny A, Dick K, et al. Peristeen integrated transanal irrigation system successfully treats faecal incontinence in children. J Pediatr Urol 2014;10:219-22.

31. Soh HJ, Nataraja RM, Pacilli M. Prevention and management of recurrent postoperative Hirschsprung's disease obstructive symptoms and enterocolitis: Systematic review and meta-analysis. J Pediatr Surg 2018;53:2423-9.

32. Demehri FR, Halaweish IF, Coran AG, et al. Hirschsprungassociated enterocolitis: pathogenesis, treatment and prevention. Pediatr Surg Int 2013;29:873-81.

33. Demehri FR, Frykman PK, Cheng Z, et al. Altered fecal short chain fatty acid composition in children with a history of Hirschsprung-associated enterocolitis. J Pediatr Surg 2016;51:81-6.

34. Gosain A, Frykman PK, Cowles RA, et al. Guidelines for the diagnosis and management of Hirschsprung-associated enterocolitis. Pediatr Surg Int 2017;33:517-21.

35. Kapur RP, Smith C, Ambartsumyan L. Postoperative Pullthrough Obstruction in Hirschsprung Disease: Etiologies and Diagnosis. Pediatr Dev Pathol 2020;23:40-59.

36. Kapur RP, Reyes-Mugica M. Intestinal neuronal dysplasia type B an updated review of a problematic diagnosis. Arch Pathol Lab Med 2019;143:235-43.

37. Mueller CM, Beaunoyer M, St-Vil D. Topical mitomycin-C for the treatment of anal stricture. J Pediatr Surg 2010;45:241-4.

38. Langer JC. Repeat pull-through surgery for complicated Hirschsprung's disease: Indications, techniques, and results. J Pediatr Surg 1999;34:1136-41.

39. Wildhaber BE, Pakarinen M, Rintala RJ, et al. Posterior myotomy/myectomy for persistent stooling problems in Hirschsprung's disease. J Pediatr Surg 2004;39:920-6.

40. Nah SA, de Coppi P, Kiely EM, et al. Duhamel pullthrough for Hirschsprung disease: a comparison of open and laparoscopic techniques. J Pediatr Surg 2012;47:308-12.

41. Chatoorgoon K, Pena A, Lawal TA, et al. The problematic Duhamel pouch in Hirschsprung's disease: Manifestations 
and treatment. Eur J Pediatr Surg 2011;21:366-9.

42. Sun S, Chen G, Zheng S, et al. Usefulness of posterior sagittal anorectoplasty for redo pull-through in complicated and recurrent Hirschsprung disease: Experience with a single surgical group. J Pediatr Surg 2017;52:458-62.

43. Coe A, Collins MH, Lawal T, et al. Reoperation for Hirschsprung disease: Pathology of the resected problematic distal pull-through. Pediatr Dev Pathol 2012;15:30-8.

44. Han JW, Youn JK, Oh C, et al. Why Do the Patients with Hirschsprung Disease Get Redo Pull-Through Operation? Eur J Pediatr Surg 2019;29:431-6.

45. Friedmacher F, Puri P. Residual aganglionosis after pullthrough operation for Hirschsprung's disease: a systematic review and meta-analysis. Pediatr Surg Int 2011;27:1053-7.

46. Jiang M, Li CL, Cao GQ, et al. Laparoscopic Redo PullThrough for Hirschsprung Disease Due to Innervation Disorders. J Laparoendosc Adv Surg Tech A 2019;29:424-9.

47. Veras LV, Arnold M, Avansino JR, et al. Guidelines for synoptic reporting of surgery and pathology in Hirschsprung disease. J Pediatr Surg 2019;54:2017-23.

48. Kobayashi H, Hirakawa H, Puri P. Abnormal internal anal sphincter innervation in patients with Hirschsprung's disease and allied disorders. J Pediatr Surg 1996;31:794-9.

49. Roorda D, Abeln ZA, Oosterlaan J, et al. Botulinum toxin injections after surgery for Hirschsprung disease: Systematic review and meta-analysis. World J Gastroenterol 2019;25:3268-80.

50. Church JT, Gadepalli SK, Talishinsky T, et al. Ultrasoundguided intrasphincteric botulinum toxin injection relieves obstructive defecation due to Hirschsprung's disease and internal anal sphincter achalasia. J Pediatr Surg 2017;52:74-8.

51. Millar AJW, Steinberg RM, Raad J, et al. Anal Achalasia After Pull-Through Operations for Hirschsprung's Disease - Preliminary Experience with Topical Nitric Oxide. Eur J Pediatr Surg 2002;12:207-11.

52. Friedmacher F, Puri P. Comparison of posterior internal anal sphincter myectomy and intrasphincteric botulinum toxin injection for treatment of internal anal sphincter achalasia: a meta-analysis. Pediatr Surg Int 2012;28:765-71.

53. Heikkinen M, Lindahl H, Rintala RJ. Long-term outcome after internal sphincter myectomy for internal sphincter achalasia. Pediatr Surg Int 2005;21:84-7.

54. Zaslavsky C, Reverbel da Silveira T, Maguilnik I. Total and Segmental Colonic Transit Time with Radio-Opaque
Markers in Adolescents with Functional Constipation. J

Pediatr Gastroenterol Nutr 1998;27:138-42.

55. Southwell BR, Clarke MCC, Sutcliffe J, et al. Colonic transit studies: normal values for adults and children with comparison of radiological and scintigraphic methods. Pediatr Surg Int 2009;25:559-72.

56. Rodriguez L, Heinz N, Nurko S. Utility of Colon Manometry in Guiding Therapy and Predicting Need for Surgery in Children With Defecation Disorders. J Pediatr Gastroenterol Nutr 2020;70:232-7.

57. Roorda D, Surridge TJ, Visschers RGJ, et al. Redo surgery with longitudinal resection for dilated bowel in Hirschsprung disease: an illustrative case series. Int J Colorectal Dis 2019;34:1983-7.

58. Ladi-Seyedian SS, Sharifi-Rad L, Manouchehri N, et al. A comparative study of transcutaneous interferential electrical stimulation plus behavioral therapy and behavioral therapy alone on constipation in postoperative Hirschsprung disease children. J Pediatr Surg 2017;52:177-83.

59. Sulkowski JP, Nacion KM, Deans KJ, et al. Sacral nerve stimulation: A promising therapy for fecal and urinary incontinence and constipation in children. J Pediatr Surg 2015;50:1644-7.

60. Lagares-Tena L, Millán-Paredes L, Lázaro-García L, et al. Sacral neuromodulation in patients with congenital faecal incontinence. Special issues and review of the literature. Tech Coloproctol 2018;22:89-95.

61. Alekseev M, Rybakov E, Shelygin Y, et al. A study investigating the perfusion of colorectal anastomoses using fluorescence angiography: results of the FLAG randomized trial. Colorectal Dis 2020;22:1147-53.

62. Van Leeuwen K, Teitelbaum DH, Elhalaby EA, et al. Long-term follow-up of redo pull-through procedures for Hirschsprung's disease: Efficacy of the endorectal pullthrough. J Pediatr Surg 2000;35:829-33; discussion 833-4.

63. Dingemans A, van der Steeg HJJ, Rassouli-Kirchmeier $\mathrm{R}$, et al. Redo pull-through surgery in Hirschsprung disease: Short-term clinical outcome. J Pediatr Surg 2017;52:1446-50.

doi: $10.21037 / \operatorname{tgh}-20-224$

Cite this article as: Demehri FR, Dickie BH. Reoperative techniques and management in Hirschsprung disease: a narrative review. Transl Gastroenterol Hepatol 2021;6:42. 